\title{
Unlocking the intraspecific aquaculture potential from the wild biodiversity to facilitate aquaculture development
}

Short title: Aquaculture potentials within species

\author{
Lola Toomey ${ }^{1}$, Pascal Fontaine ${ }^{1}$, Thomas Lecocq ${ }^{1, *}$ \\ ${ }^{1}$ Université de Lorraine, INRAE, URAFPA, F-54000 Nancy, France \\ * Corresponding author: \\ thomas.lecocq@univ-lorraine.fr \\ University of Lorraine, Boulevard des Aiguillettes, BP 70239, 54506 Vandœuvre-lès-Nancy, France
}

\begin{abstract}
Domestication of new candidate species remains a long and difficult process often resulting in unfruitful attempts. Early steps in most domestication programs often consider species as a unity, disregarding a part of the biodiversity: the wild intraspecific geographic differentiation. Yet, this differentiation can shape local specificities, which could lead to different domestication predisposition or socio-economic attractiveness between populations. Therefore, considering this population-specific potential could facilitate domestication and subsequent production of new candidate species. Here, we propose a three-step integrative approach to standardize and facilitate new domestication attempts by taking advantage of wild geographic differentiation. The step 1 consists in classifying the wild biodiversity to identify prospective units (i.e. groups of differentiated allopatric populations). The step 2 allows comparing performances of these units in standardized conditions (i.e. rearing system) through a multifunction and multi-trait assessment. Finally, the step 3 highlights units with higher aquaculture potentials through the calculation of an aquaculture potential score. This approach, here applied to fish culture for human consumption, also aims at being extended to other taxa (e.g. crustaceans, molluscs) and other production goals.
\end{abstract}

Keywords: Domestication, Fish culture, Intraspecific differentiation, Multi-function, Multitrait, Sustainability 


\section{CONTEXT}

Aquaculture is the farming of aquatic organisms through several alternative practices (i.e. monoculture/polyculture, outdoor/indoor, flow through systems/recirculated systems, and extensive/intensive). Since the 1960s, aquaculture production has rapidly expanded and exponentially grown worldwide as far as to provide more than $50 \%$ of the world's aquatic food consumption nowadays (FAO 2018). This development has been triggered by the need to meet the increasing human demand for aquatic products since wild fisheries catches are no longer sufficient (Watson and Pauly 2001; Froese et al. 2012). Nevertheless, aquaculture is often criticized due to its negative environmental impacts and its potential unsustainable development (Martinez-Porchas and Martinez-Cordova 2012; Christou et al. 2013; Diana et al. 2013). The low species diversity which contributes to worldwide aquaculture production is one of the main causes of these issues. Indeed, although numerous species have been farmed, the production of most of these species (i) was quickly abandoned due to zootechnical or economic problems or (ii) remains marginal when compared to the total volume of aquaculture production (Teletchea and Fontaine 2014; Metian et al. 2019). Thereby, $85 \%$ of the current world fish production relies on about fifteen species (Lazard 2013). Such production model based on few species, jeopardizes (i) local native fish fauna by increasing interspecific competition in the wild (Garibaldi and Bartley 1998; Silva et al. 2009) or triggering pathogen spillover (Martinez-Porchas and Martinez-Cordova 2012) when accidental introductions or invasions by the alien farmed species happen, (ii) human food security since the heavy dependence on few species puts at risk aquaculture production (e.g. if an epizootic outbreak happens in farmed species, see for instance Godoy et al. 2008), and (iii) economic prospect since poorly diversified production limits the adaptive potential of aquaculture to face environment/consumer demand changes (Fontaine 2009; Metian et al. 2019). In face of such threats, international organizations (e.g. the Food and Agriculture Organization of the United Nations) are strongly advocating for diversification in regard to species while strengthening wellestablished species (FAO 2018; Metian et al. 2019). Indeed, species diversification appears as one way to improve the sustainability of the aquaculture sector and promote its resilience, particularly through the production of new species occupying other market segments, for instance niche markets (Liao and Huang 2000; Muir 2005; Fontaine et al. 2009; FAO 2018; Metian et al. 2019). This political will has triggered the development of new species production (e.g. DIVERSIFY project [https://www.diversifyfish.eu/], Aruho et al. 2018; Mitra et al. 2018). 
One of the biggest challenges in aquaculture diversification is the required domestication of wild taxa (Liao and Huang 2000; Harache 2002; Fontaine et al. 2009). Domestication is the process in which some populations are bred in a human-controlled environment and modified across successive generations from their wild ancestors in ways making them more useful to humans who control increasingly during the process their reproduction and food supply (Lecocq 2019). This is a stepwise process (i.e. the domestication levels sensu Teletchea and Fontaine 2014) ranging from the first trials of acclimatization (level one) in the culture environment to the life cycle completion in captivity (levels three and four) and, eventually, to the selective breeding program setting up (level five). It is a long and tough process and attempts are often unfruitful (Teletchea and Fontaine 2014). Indeed, previous domestication attempts on new species show that such tries often lasted only a few years before being abandoned (Teletchea and Fontaine 2014; Metian et al. 2019). Furthermore, even when the life cycle is completed in captivity, major bottlenecks can still remain, impeding domestication continuation and production development (Teletchea and Fontaine 2014). Such failures and issues result from the inability of domestication programs to meet challenges of initiating domestication process (i.e. acclimatization), completing lifecycle, and overcoming major bottlenecks (e.g. very low survival rate) due to technical limitations, economic constraints, or some intrinsic species traits (i.e. single feature or quantifiable measurement of an organism; Jobling 2010). These latter are for instance low growth rate, sensitivity to disturbances or diseases, or high aggressiveness, which characterize poor candidates for domestication (Liao and Huang 2000; Jobling 2010). In contrast, most of already domesticated and fruitfully produced species display the opposite expression pattern for these traits (Diamond 2002). Other intrinsic features can also be important in some particular human-controlled environments such as the ability to tolerate high rearing densities in intensive culture (Jobling 2010) or the resistance to environmental disturbances occurring in outdoor aquaculture (Ficke et al. 2007). This means that some species might be suitable for a particular rearing system but would poorly perform in another. Overall, a successful domestication requires the favorable expression of traits involved in several biological functions or intrinsic characteristics (i.e. trait not associated to a specific biological function) of the targeted species. Indeed, a group of individuals can be domesticated if it can reproduce (reproductive and behavior functions), grow (growth, development, and behavior functions), feed (nutrition and behavior functions), and overcome stresses (e.g. resistance to diseases, immune, and behavior functions) in a particular controlled environment. Besides these traits, the costly domestication 
process of a wild taxon cannot be achieved without stakeholders' support and/or interest (Suquet 2010), highlighting the importance of considering other traits involved in the socio-economic context (e.g. product quality traits). Overall, all of these factors shape the so called aquaculture potential: a quantification of how much expression of all key traits/functions (biological and socioeconomic traits) is favorable for domestication and subsequent production. Therefore, the aquaculture potential assessment requires a multi-function and a multi-trait evaluation. Beside the species intrinsic aquaculture potential, domestication of a taxon is unlikely if the human needs that it could meet are already addressed (e.g. by other currently domesticated species [Le François et al. 2010] or when capture fisheries already fulfil consumer demand for a particular species, e.g. Otterå 2004).

The differential species suitability for domestication programs places a premium on the development of an approach to find relevant candidates in the wild biodiversity. High-throughput screening of the wild biodiversity has recently been proposed as a solution to highlight such species in crops by testing many wild candidate taxa (DeHaan et al. 2016). These candidates are tested across several steps including a first step of screening wild species to identify candidates of interest through an evaluation of traits linked to several biological functions (e.g. growth rate, yield, suitability for special diets; DeHaan et al. 2016). Similar approaches, although less developed, are also available for fishes and consider wild fish biological characteristics as well as socio-economic traits in the evaluation process (e.g. Le François et al. 2002; Quéméner et al. 2002). Nevertheless, such approaches often consider species as a unity and disregard wild inter-populational geographic differentiation (sensu divergence between allopatric groups of conspecifics; Mayr 1963) and its consequences on aquaculture potential.

\section{WILD INTRASPECIFIC DIFFERENTIATION: AN ASSET FOR FISH FARMING?}

Inter-populational geographic differentiation is a common phenomenon in fish species (see Bernatchez and Wilson 1998; Costedoat and Gilles 2009) which could be important for new domestication programs. Indeed, allopatric populations can undergo specific demographic histories as well as geographic and ecologic isolation triggering (i) gene flow disruption or limitation and subsequently genetic divergence and (ii) local adaptations to different selection regimes (Mayr 1963; Avise 2000). Ultimately, this shapes local specificities in genetic, morphology, physiology, 
behavior, and/or ecology which could impact the aquaculture potential and economic attractiveness of each wild population (e.g. for non-fish species in Lecocq et al. 2016a,b).

In non-fish species, geographic differentiation of important traits for domestication and production is known for several wild species of interest such as plants (e.g. Ben Mansour-Gueddes et al. 2010; Healey 2016) and fungi (e.g. Liti et al. 2009; Warringer et al. 2011). Moreover, a few previous success stories show that the best candidates to start new species production are not a particular species but rather one or some particular groups of populations within the species of concern for a particular culture system. The bumblebee production industry provides one remarkable example where population-specificity inclusion through multi-trait evaluation in domestication programs fostered a fruitful economic development (Velthuis and van Doorn 2006; Lecocq et al. 2016a,b). One species, the buff-tailed bumblebee (Bombus terrestris) displays significant differentiation in key traits for bombiculture (i.e. foraging efficiency, behavior, colony size, diapause condition, and phenology) between allopatric groups of populations corresponding to subspecies (reviewed in Lecocq et al. 2016b). In the early years of production, European bumblebee breeders tried to domesticate several subspecies (Velthuis and van Doorn 2006). Within a fairly short amount of time, one of these subspecies proved to have superior characteristics from a commercial point of view and has become the dominant taxa in the bumblebee production industry (Lecocq et al. 2016b).

In fish production, geographic differentiation has been poorly considered at the beginning of new wild fish domestication trials. However, the few examples involve some of the most successful fish productions. In the 1970s, 41 wild Scandinavian salmon (Salmo salar) populations were bred in a standardized production environment to evaluate their performances (Gjedrem et al. 1991). This work considered, on different year classes, several desirable traits (e.g. growth, age at sexual maturity, furunculosis resistance, filet color) in order to identify the best populations to be used for production (which greatly improved salmon production, Gunnes and Gjedrem 1978, Gjedrem et al. 1991; Gjedrem 2010, 2012). Later, in the 1990s, four Middle Eastern and Asian farmed populations along with four wild allopatric African populations of Nile tilapia (Oreochromis niloticus) were crossbred to produce fish stocks (Eknath et al. 1993; Gjedrem 2012). Among these fish, best breeders (i.e. with highest growth rate) were selected to develop the genetically improved 
farmed tilapia strain that grows up to $85 \%$ faster than the earlier farmed fish stocks and is now disseminated across the world (Gjedrem 2012).

Conversely to the bumblebee example, the Scandinavian salmon and Nile tilapia cases only show how the geographic differentiation can be useful for advanced fish domestication programs. Here, we argue that fish domestication programs could take advantage of geographic differentiation to initiate or to continue the earlier levels of the domestication process (i.e. levels from one to three, and possibly four, see levels in Teletchea and Fontaine 2014). Indeed, non-domesticated or species at incipient domestication levels can display, when bred in captive conditions, differentiation for several traits impacting the aquaculture potential such as features related to growth (e.g. Vanina et al. 2019a), nutrition (e.g. Jonassen et al. 2000), reproduction (e.g. Kokita 2004), aggressiveness (e.g. Toomey et al. 2019), immunity (e.g. Glover et al. 2003), and stress sensibility (e.g. Delabbio et al. 1990). Therefore, considering wild geographic differentiation when starting new fish domestication programs could unlock population-specific potential included in the wild intraspecific biodiversity.

Besides the obvious choice of populations with the highest aquaculture potential to start domestication programs, differentiated populations can display specificities that could allow overcoming current bottlenecks in species at intermediate domestication levels (i.e. level four; e.g. the aquaculture of Perca fluviatilis, a species facing strong bottlenecks in its development due to a low larval survival rate and high deformity rate; Fontaine and Teletchea 2019). In such species, assessment of wild population aquaculture potential could pave the way to improve current fish stock or even restart an improved domestication process with better populations if no alternatives can be considered.

Since considering wild geographic differentiation could facilitate aquaculture diversification process, it raises the question how its integration in domestication programs should be achieved. Indeed, this integration cannot be based on very long-term or unrationalized experiments otherwise using wild geographic differentiation for domestication programs would be poorly justified. Therefore, we here propose a conceptual approach to facilitate the integration of geographic differentiation in new candidate species domestication processes. 


\section{HOW TO TAKE INTO ACCOUNT WILD GEOGRAPHIC DIFFERENTIATION: A THREE-STEP INTEGRATIVE APPROACH}

The aim of this work is to propose a new standardized and rationalized three-step integrative approach to integrate wild intraspecific differentiation in new domestication programs through a multi-function and multi-trait assessment. This approach aims at facilitating domestication of new wild fish candidates or species at incipient domestication levels facing major bottlenecks in the domestication process and/or production-related issues. It aims at, successively, classifying, evaluating, and identifying best population group(s) in the context of new domestication trials (Fig. 1). 


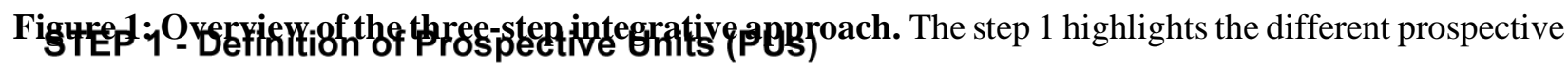

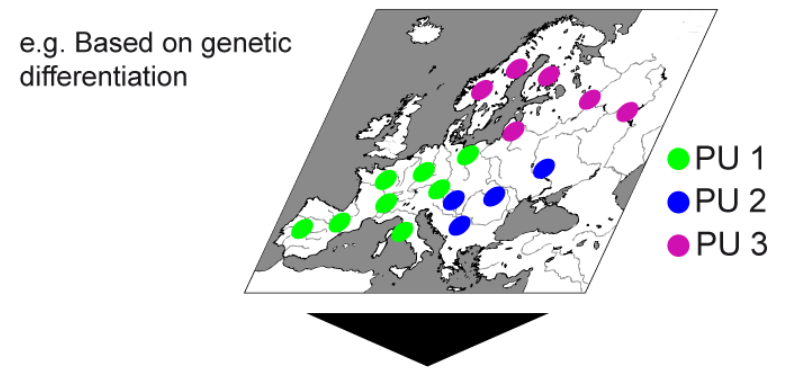

STEP 2 - Testing divergences of performances of PUs in aquaculture conditions

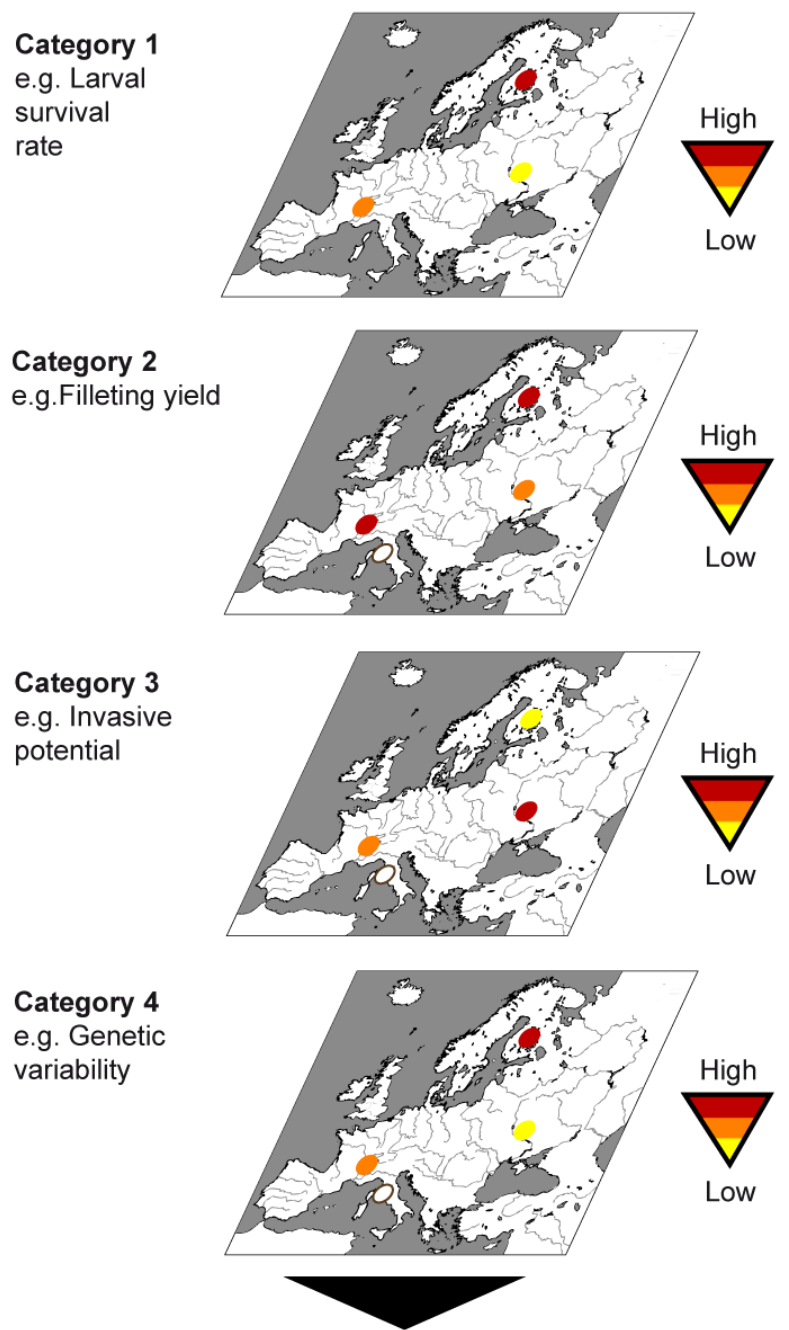

STEP 3 - Identification of units with high aquaculture potential

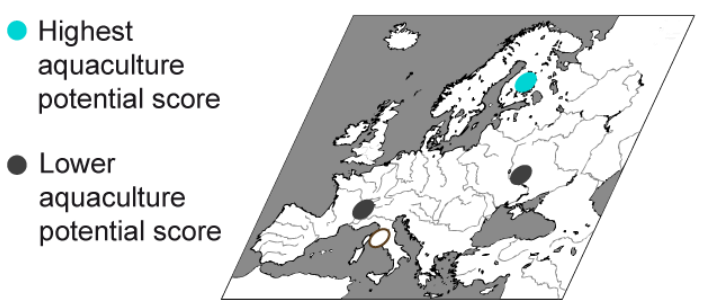


units (PU). A population representative of the prospective unit is sampled between steps 1 and 2. Category 1 includes biological traits measured on a group of individuals of the target prospective unit which reflect the fitness (i.e. ability to survive, grow and reproduce) in captive conditions. Category 2 includes biological traits which are specifically relevant to a particular production system (e.g. RAS, pond, raceway) and/or a specific production goal (e.g. fillet, whole fish sale). Category 3 includes biological traits linked to the interaction between the group of individuals and the rearing system which could impact surrounding environments. Category 4 includes biological traits relative to future major challenges and disturbances. In order to simplify the figure, only one trait is represented for each category in the step 2.

\section{Step 1 - Classification of the wild intraspecific biodiversity}

Evaluating the potential for aquaculture of all wild populations of a particular species would be time/money-consuming and is, ultimately, not achievable in practice. Moreover, such comprehensive evaluation would be inaccurate because all conspecific populations are not genetically and phenotypically divergent (Mayr 1963; Hewitt 2000). However, comparing randomly chosen wild populations is not an efficient solution to this issue because the best population(s) for aquaculture purpose could be missed out. An efficient rationalization relies on the development of a classification system that can identify population groups that have different/independent demographic histories and/or potential local adaptations, making divergences in aquaculture potentials likely. Therefore, such a classification should be based on proxies reflecting evolutionary histories and/or local adaptations of the different population groups. Three different proxies can be used: (i) phenotype, (ii) habitat, and (iii) genetic information.

Phenotype, especially morphological characteristics (e.g. color patterns, shape), is the earliest, the easiest, and the most commonly used information to classify biological units (Strauss and Bond 1990; Haig et al. 2006; Craig et al. 2017). It has been widely used to define subspecies (Mayr 1963; Haig et al. 2006) which can be an efficient classification strategy to split population groups with different aquaculture potentials (e.g. for fish species, Pivnička 1970; Karakousis et al. 1991; Kalayci et al. 2018). Other phenotypic information such as reproductive or behavior traits could be used but, to our knowledge, they have not been applied in fish species to classify at the intraspecific level. Nevertheless, phenotypic traits can be unreliable since there can be a part of subjectivity when defining and interpreting character states (Padial et al. 2010) and it often requires extensive 
investigation of inter-individual variability (Haig et al. 2006). Moreover, some phenotypic traits can be plastic and might not reflect population-specific histories or local adaptations (Vogler and Desalle 1994; Joyce et al. 2009). These concerns have led to criticisms regarding the relevance and the efficiency of phenotype-based intraspecific classifications (e.g. Mallet 2001; Patten 2010). Although phenotype information could still be useful under the condition that trait variations are indicative of underlying genetic divergences (e.g. morphology in brown trout [Salmo trutta]; Pakkasmaa and Piironen 2001; Kalayci et al. 2018), such classifications must be applied prudently.

Habitat information (biotic and/or abiotic factors) has rarely been used to classify conspecific populations (but see e.g. the distinction between habitats, such as rivers or lakes, contributed to define intraspecific units in S. trutta, Blanc et al. 1971; Guyomard 1989). Yet, one could expect that populations living in different biotic/abiotic conditions can display different demographic histories or local adaptations (Avise 2000). Therefore, a relevant approach to classify populations before evaluating their aquaculture potential could simply consist of developing a classification based on habitat differentiations (e.g. different biogeographic regions, distinct hydrographic basins, different water parameters). However, establishing if different habitats can lead to differential evolutionary paths between allopatric populations is still hard to achieve due to the potential noncongruence with population-specific histories and/or local adaptations. Moreover, there is a part of subjectivity when selecting criteria to detect habitat specificity. Therefore, these limitations make this classification approach unpractical to date for fishes.

Genetic information has been increasingly taken into account to investigate intraspecific differentiation (Frankham et al. 2004; Padial et al. 2010). The phylogeography-based classification appears as one of the most suitable strategies to achieve an a priori classification. Phylogeography aims at identifying groups of genetically differentiated populations which underwent specific demographic histories (Avise 2000) and could have acquired, through local adaptations, some phenotypic specificities. Moreover, information about phylogeographic structure is already available for many fish species (Bernatchez and Wilson 1998; Costedoat and Gilles 2009). In the context of our three-step approach, genetic proxies allow maximizing chances of detecting populations with different potentials for aquaculture that can be conserved in production environments. Indeed, domesticated fishes will often be produced in a culture environment which can be quite different from their native habitat. Therefore, only genetic-based divergences are of 
interest while differentiations triggered by phenotypic plasticity (i.e. the ability of a genotype to vary in phenotype depending on environmental conditions; Pigliucci et al. 2006) processes would be useless. However, special attention should be paid to the limitations of the genetic approach. On the one hand, an approach using only few arbitrarily picked genetic pieces of evidence could result in an insufficient resolution to detect populations with different potentials for aquaculture because phenotypic or ecologic differentiations are not always being represented by many genetic differences, making their detection unlikely when using a small part of the genome (Arif and Khan 2009; Cruaud et al. 2014; Patwardhan et al. 2014). This places a premium on using large genome parts or highly variable DNA sequences. On the other hand, a high resolution genetic-based classification could fail because of absence of interpopulational variation of the potential for aquaculture despite different evolutionary histories revealed by genetic markers. Using sequences of genes involved in traits impacting the potential for aquaculture could be an efficient solution. Unfortunately, such an approach remains unfeasible to date because (i) most traits are under polygenic control, (ii) the genetic basis of variation in traits is often very complex, and (iii) quantitative trait loci are mostly studied for a few major aquaculture species (Falconer and Mackay 1996; Yue 2014; Abdelrahman et al. 2017; Robledo et al. 2018). Therefore, the risk of using a classification which might not lead to the identification of inter-populational divergences cannot be ruled out to date but several studies already highlighted a congruence between genetic differentiation in neutral markers and phenotypic differentiation in controlled conditions (e.g. Rosauer et al. 2011; Vanina et al. 2019a).

Overall, special attention should be paid to the a priori classification used, and advantages/disadvantages of each proxy should be assessed for each target species through coworking with systematicians, phylogeographers, and ecologists. The resulting classification allows identifying the different prospective units (i.e. groups of allopatric populations differentiated) that will be evaluated in the step 2 .

\section{$\underline{\text { Step } 2 \text { - Evaluating keys traits of the aquaculture potential }}$}

The step 2 aims at comparing the performances of prospective units in fish farming conditions through an integrative (i.e. multi-function and multi-trait) assessment. For a wild species for which even acclimatization has not been tried before, it implies to first design a culture system that most likely will be suitable for the targeted species and stakeholders. Such a system can be defined by 
applying farming conditions used for phylogenetically closely related or ecologically similar fish species that are already produced in captivity or using knowledge from habitat conditions experienced by wild populations (Liao and Huang 2000; Teletchea et al. 2009). For fish species for which incipient production already exists, the current aquaculture conditions should be used. Choosing the aquaculture system (i.e. domestication system) in which wild populations will be assessed is an important preliminary step. Indeed, expression of traits shaping the aquaculture potential can change according to the environment (e.g. Rowland et al. 2004). A relevant aquaculture potential assessment should be thus obtained through bioassays in a human-controlled environment as close as possible to the targeted production system (e.g. cages, flow-through system, indoor Recirculated Aquaculture System [RAS], ponds). This means that all prospective units should be evaluated in a common-garden experiment in which the rearing system is similar to future rearing conditions. Therefore, this evaluation might have to be performed again if another rearing system is considered (i.e. the results from a RAS assessment might not be useful for pond production).

Special attention should be paid to which set of individuals is needed for the aquaculture potential assessment. Two alternative solutions can be used: (i) broodstock sampling or (ii) early-life stage collection. One could consider that it is necessary to sample wild broodstock to produce captiveborn offspring that will be evaluated. This requires respecting good rearing practices in stock constitution such as using a sufficient number of individuals in order to avoid inbreeding issues (relatively to the minimal effective size; Duncan et al. 2013). However, sampling broodstock could be difficult for some species because tolerance to transport and acclimatization varies between fish taxa depending on the life stage considered (Berka 1986; Omeji et al. 2017, e.g. for Perca fluviatilis, early life stages (i.e. eggs, juveniles) remain the preferred wild biological material transported by fish farmers given the high adult mortalities subsequent to transport). This implies that the sampling material choice can be species-dependent. Therefore, for some species, early life stages could be sampled as initial biological material when accessible, but the phenotypic plasticity impact might be more substantial. Beside the choice of life stage to be sampled, it should be noted that, although the step 1 aims at defining groups with similar demographic histories and/or potential local adaptations and, thus, similar aquaculture potentials, different populations from the same prospective unit could display different performances. Indeed, temporary specific environmental disturbances affecting some populations of the same prospective units (e.g. unusual regional food 
shortage, disease outbreak, or local pollution events) can impact evaluated traits (e.g. Scott and Sloman 2004; Claireaux et al. 2013). In such case, the initial state of the sampled population impacts results obtained in controlled conditions (e.g. 100\% mortality if presence of Perhabdovirus in Percids; Bigarré et al. 2017; Rupp et al. 2019). Consequently, a reliable evaluation of prospective unit aquaculture potential could include an assessment of the initial state of wild sampled populations through, for instance, toxicological (e.g. Tabari et al. 2010) and veterinary tests (e.g. Talbi et al. 2011; Bigarré et al. 2017) as well as a check-up of the quality of breeders (e.g. lipid content in oocytes; Dhurmeea et al. 2018). Unfortunately, some of such tests are still unavailable (e.g. unavailability/lack of efficiency of some disease detection methods) or unfeasible at large scale. Therefore, two strategies could be used: (i) sampling the same wild populations at different years and repeating independently the step 2 and/or (ii) avoiding sampling populations occurring in areas where unusual events have been recently recorded (e.g. recent chemical pollution). Because the former strategy would increase the workload, we regard this strategy as poorly implementable and recommend the latter.

The suitability of traits chosen for the evaluation is based on the relevance of the trait (i.e. integrative traits, important to measure and relevant regarding welfare and ethics) and its accessibility (i.e. trait measurable at large scale but not necessarily evaluated yet in routine because of the lack of proper method). A literature overview of such traits for fishes (e.g. Lensi 1995; Liao and Huang 2000; Quéméner et al. 2002; Otton 2004; Teletchea et al. 2009; Jobling 2010; Le François et al. 2010) allowed us to select a first set of traits which are overall relevant for teleosts (Table 1). This selection has been further reinforced by a survey conducted among Percids and Sturgeons producers. These two species groups were chosen since they include species with recent domestication processes and with different production outputs (see Annex S1). The ongoing production of these species allows having an overview on traits which turned out to be essential for production. We asked farmers (ongoing production of one or both species groups) in this survey to give their opinion on the different traits selected and to suggest additional relevant traits to be considered (see details and results in Annex S1 and Fig. S1). For all traits involved in the aquaculture potential assessment, there is a performance expected by stakeholders (e.g. growth: expected high growth rate; deformity: low deformity rate). Traits chosen were classified in four categories (Table 1) corresponding to the different aspects which need to be considered when starting a new domestication process. 
The first category is characterized by all biological traits measured on a group of individuals of the target prospective unit which reflect the fitness (i.e. ability to survive, grow, and reproduce) in captive conditions (Table 1). This category is common to all rearing systems (e.g. on-growing survival rate important in all production systems when on-growing is part of the production outlet). It includes traits linked to the different steps of a production cycle (reproduction, larviculture, ongrowing) but also to the different biological functions (reproduction, growth, nutrition, and behavior).

The second category is characterized by all biological traits which are specifically relevant to a production system (e.g. RAS, ponds, floating cages) and/or a specific production goal (e.g. fillet, whole fish sale). It includes traits (i) involved in responses to breeding-environment-specific stresses (e.g. antipredator behavior is relevant in ponds [Huntingford et al. 2012] while resistance to handling is important in intensive monoculture in RAS, Milla et al. 2010) and (ii) related to the production target (e.g. filleting yield for the food consumption output). Here, we consider as examples two different scenarios with a common outlet (consumption market) but different rearing systems commonly used in aquaculture: RAS and ponds (Table 1). 
Table 1: Set of traits considered in the integrative approach in the context of monoculture in RAS and ponds for the consumption market. Category 1 includes biological traits measured on a group of individuals of the target prospective unit which reflect the fitness (i.e. ability to survive, grow and reproduce) in captive conditions. Category 2 includes biological traits which are specifically relevant to a production system (e.g. RAS, pond, raceway) and/or a specific production goal (e.g. fillet, whole fish sale). Category 3 includes biological traits linked to the interaction between the group of individuals and the rearing system, which could impact surrounding environments. Category 4 includes biological traits relative to future major challenges and disturbances. Traits in italic correspond to traits less important according to Percid and Sturgeon farmers (less than $50 \%$ of opinions considering the trait as quite important or important for at least one or both species groups).

\begin{tabular}{|c|c|c|c|}
\hline Category & Sub-category & Trait group & Trait \\
\hline & \multirow{13}{*}{ Reproduction } & & Breeder survival rate \\
\hline & & Breeders & Age and size at sexual maturity \\
\hline & & & Fecundity \\
\hline & & & Volume of sperm \\
\hline & & Gametes & Number of oocytes \\
\hline & & & Number of spermatozoids \\
\hline & & & Survival rate \\
\hline & & & Egg diameter \\
\hline & & Fertilized eggs & Malformation rate \\
\hline \multirow[t]{9}{*}{ Category 1} & & & Fertilization rate \\
\hline & & & Hatching rate \\
\hline & & & Spawning timing \\
\hline & & & Spawning duration \\
\hline & \multirow{5}{*}{ Larviculture } & & Growth rate \\
\hline & & & Growth heterogeneity \\
\hline & & & Volume of the yolk sac \\
\hline & & Nutrition & Larvae size at hatching \\
\hline & & & $\begin{array}{c}\text { Timing for the exogenous feeding } \\
\text { transition }\end{array}$ \\
\hline
\end{tabular}


Swim bladder inflation rate

Development Deformity rate

Survival rate

\section{Cannibalism rate}

Aggressive behavior Age at cannibalism onset

Size at cannibalism onset

Growth rate

Growth

Growth heterogeneity

On-growing

Development
Sex-ratio

Survival rate
Nutrition

\section{Food conversion ratio}

\begin{tabular}{|c|c|c|c|}
\hline \multirow{8}{*}{ Category 2} & \multirow{7}{*}{$\begin{array}{l}\text { Intensive } \\
\text { monoculture } \\
\text { in RAS - } \\
\text { consumption } \\
\text { market }\end{array}$} & \multirow{3}{*}{$\begin{array}{c}\text { Traits involved in response } \\
\text { to captive environment } \\
\text { pressures }\end{array}$} & $\begin{array}{c}\text { Resistance to sub-lethal stress and } \\
\text { diseases/parasites which are } \\
\text { expectable in RAS environment } \\
\text { (e.g, handling) }\end{array}$ \\
\hline & & & $\begin{array}{c}\text { Acceptance of artificial diet } \\
\text { (weaning) } \\
\text { Group structure }\end{array}$ \\
\hline & & & $\begin{array}{c}\text { Activity (i.e. individuals more or } \\
\text { less active) } \\
\text { Water volume occupation }\end{array}$ \\
\hline & & \multirow{4}{*}{$\begin{array}{l}\text { Traits important for output } \\
\text { quality }\end{array}$} & Filleting yield \\
\hline & & & Condition factor \\
\hline & & & $\begin{array}{c}\text { Body shape } \\
\text { Organoleptic characteristics }\end{array}$ \\
\hline & & & Nutritional value \\
\hline & $\begin{array}{c}\text { Extensive } \\
\text { monoculture } \\
\text { in ponds - } \\
\text { consumption } \\
\text { market }\end{array}$ & $\begin{array}{c}\text { Traits involved in response } \\
\text { to captive environment } \\
\text { pressures }\end{array}$ & $\begin{array}{l}\text { Resistance to sub-lethal stress and } \\
\text { diseases/parasites which are } \\
\text { expectable in the pond } \\
\text { environment (e.g, physico- } \\
\text { chemical changes, transport) }\end{array}$ \\
\hline
\end{tabular}




\begin{tabular}{|c|c|c|}
\hline & & $\begin{array}{c}\text { Antipredator behavior (e.g. birds, } \\
\text { parasites) }\end{array}$ \\
\hline & & Filleting yield \\
\hline & Traits important for output & Condition factor \\
\hline & quality & Body shape \\
\hline & & Nutritional value \\
\hline & Invasive risk & Invasive potential \\
\hline Cateoorv 3 & $\begin{array}{l}\text { Pathogen spillover risk to } \\
\text { wild populations }\end{array}$ & Pathogen load \\
\hline & $\begin{array}{c}\text { Risks of genetic } \\
\text { homogenization and } \\
\text { outbreeding }\end{array}$ & $\begin{array}{c}\text { Genetic divergence between } \\
\text { farmed and neighboring wild } \\
\text { populations }\end{array}$ \\
\hline & $\begin{array}{l}\text { Maintenance of fish } \\
\text { performances in the future }\end{array}$ & $\begin{array}{c}\text { Robustness (including adaptive } \\
\text { potential to ongoing climate } \\
\text { change) }\end{array}$ \\
\hline & & $\begin{array}{c}\text { Future invasive potential in the } \\
\text { climate change prospect }\end{array}$ \\
\hline & $\begin{array}{l}\text { Future selective breeding } \\
\text { programs }\end{array}$ & $\begin{array}{l}\text { Genetic variability (fish } \\
\text { individual specificities) }\end{array}$ \\
\hline & $\begin{array}{l}\text { Ability to face new } \\
\text { stakeholder } \\
\text { requirements/expectations }\end{array}$ & Acceptance of alternative feeds \\
\hline
\end{tabular}

The third category includes all biological traits linked to the interaction between the group of individuals and the environment of the rearing system, which could impact the surrounding environments (e.g. invasive potential, pathogen load, competition; Table 1). In all rearing systems, there are risks of escapees (particularly relevant in outdoor culture, yet risks cannot be ruled out in indoor aquaculture; Summerfelt and Vinci 2009). These escapees can trigger negative impacts which vary according to the identity of populations bred (i.e. local population, non-native population introduced in a species native area or in a new area out of the geographic species native range): (i) invasive risk (estimated by the invasive potential; Kolar 2004; Naylor et al. 2005), (ii) introduction of pathogens (evaluated through the pathogen load; Naylor et al. 2005), (iii) genetic introgression/homogenization or outbreeding with local wild populations (see for example Fraser 
et al. 2008; Bolstad et al. 2017; Wringe et al. 2018), and (iv) competition risk between domesticated and wild populations (there is no assessment protocol available for fishes [but see for other vertebrates e.g. Polo-Cavia et al. 2009]; therefore, we ruled out this last risk from the aquaculture potential evaluation).

The last category is characterized by all biological traits relative to future major challenges and disturbances. A literature overview allows identifying four main challenges. First, there are high stakes to domesticate robust individuals. Here, we describe robustness as the ability of individuals to express their production potential in a wide range of environment changes (e.g. climatic changes, feeding shifts, disease outbreaks; based on Knap 2005). This includes adaptive potential to future climate change (i.e. large-scale changes predicted related to temperature, winds, and acidification) since it will provide novel challenges (Cochrane et al. 2009), especially in outdoor aquaculture (e.g. ability to survive in aquaculture conditions despite more frequent severe weather events). Second, it is also important to consider the future invasive potential, taking into account predicted climate changes, since invasive potential is partially shaped by climatic requirements. Third, the maintenance of genetic variability during domestication has been pointed out as a major feature to promote a sustainable development of aquaculture (Liao and Huang 2000). While stock management aiming at limiting inbreeding has been proposed, the selection of units with sufficiently high genetic variability increases chances to have individual specificities of interest for future selective breeding programs (e.g. rare genes counter-selected in the wild but presenting an advantage in rearing systems; Vandeputte and Launey 2004). Finally, one major challenge relies on the acceptance of alternative aquafeeds (i.e. less rich in fish oil and fish meal) such as plantbased feeds (Naylor et al. 2009). Indeed, due to wild resources limitation, it is essential to shift towards alternative feedstuffs in order to promote sustainability of aquaculture and an interindividual variability in the ability to adapt to vegetal-based diets was already assessed for some species (e.g. Burel 2017).

\section{$\underline{\text { Step } 3 \text { - Identification of units with high aquaculture potential }}$}

The step 3 aims at identifying units with high aquaculture potential through the establishment of an integrative decisional framework. 
According to our survey, traits are perceived differently among fish farmers and are not given the same level of importance (see in Table 1; Fig. S1). For instance, growth rate during on-growing is globally considered as very important for both Percids and Sturgeons while group structure (i.e. structure of a group of individuals created by relationships between several individuals; Hinde 1976) is evaluated as less important for both species. In addition, the importance of traits also varies between species groups, production systems, and production goals (Fig. S1). For instance, sex ratio is considered as quite important or important by $71.4 \%$ of Sturgeon farmers but only by $14.6 \%$ of Percid farmers. Therefore, in order to identify units with high aquaculture potential, it is necessary to adjust the importance of the different traits through weighting coefficients. Moreover, some traits are so important (e.g. major bottlenecks preventing the completion of the life cycle) that when a minimum threshold of their required expression is not met in a unit, this latter is regarded to be void of aquaculture potential. For instance, a paltry survival or fecundity rate observed in a unit will impede further domestication programs (e.g. inability for males to fertilize eggs due to the absence of courtship behavior in Senegalese sole [Solea senegalensis]; Guzmán et al. 2008; Morais et al. 2016). Nevertheless, special attention should be paid to the definition of minimum required expression threshold needed for a trait. Indeed, although some trait expressions can constitute a major bottleneck in future production, they might not fully prevent domestication/production or depress profitability (as observed in sturgeon aquaculture where the species high socio-economic value offsets its low growing out potential and late sexual maturation). Moreover, some bottlenecks could be overcome through other alternatives such as technological improvements. Therefore, we advocate that minimum expression thresholds should be defined only for characteristics which are not surmountable at the time of the evaluation and completely prevent domestication/production.

The assessment of weighting coefficients (0 to 100; adapted from Quéméner et al. 2002; similarly to breeding goals index but for which each trait is weighted according to its socio-economic value; Chavanne et al. 2016), as well as minimal expression thresholds, to all traits needs to be performed by a panel which includes all potential interested parties. These latter include fish farmers, scientists, environmental managers, and other socio-economic stakeholders. Since this approach targets species which are new candidates for domestication, stakeholders can take decisions using (i) literature, (ii) knowledge from ongoing domestication trials and farming experiences, and/or (iii) extrapolations from closely related species when no rearing experience is available. The weight coefficient and minimal expression threshold attribution can be done through a consensus for each 
trait performed by all stakeholders (e.g. mean of the weights attributed by all stakeholders or using Quaker-based or spokescouncil models; Roy and Bouyssou 1993; Hartnett 2011). These weighting coefficients and minimal expression thresholds need to be evaluated for each new domestication attempt since they can vary between species targeted and through time depending on stakeholders' expectations.

Besides trait weighting, it is unlikely that a prospective unit has the best performances for all criteria. It is more likely that a unit displays the best performance for a specific trait (e.g. best growth rate) but the worst for another (e.g. lowest larval survival rate). Therefore, an indicator is required in order to make a synthesis at the multi-function and multi-trait levels to identify units with high aquaculture potential. Some methods and associated scores were suggested at the interspecific level in order to identify good candidate species (e.g. method used in Quéméner et al. 2002; Le François et al. 2010; Suquet 2010; Alvarez-Lajonchère and Ibarra-Castro 2013; at the intraspecific level Toomey et al. under review). These scoring methods, or other indexes available in literature (e.g. similarly to the selection index, Gjedrem 2005a), could be used to evaluate the aquaculture potential at the intraspecific level.

\section{WHAT ARE THE LIMITATIONS OF THE APPROACH?}

Overall, three limitations should be stated: (i) methodological limitations, (ii) pragmatic concerns, and (iii) legal regulation context.

\section{$\underline{\text { Methodological limitations }}$}

A first limitation can be dreaded if translocations of the targeted species happen concurrently with the three-step approach evaluation. If they occur between the steps one and two of the approach, the classification step would be irrelevant for next steps of the approach. Moreover, if they happen after the selection of units with high potential for aquaculture, future population resampling in the wild could lead to the establishment of fish stock with specificities different from those expected according to the previous three-step assessment. Even species that are not currently produced can undergo such a phenomenon because they can be the focus of recreational activities (e.g. angling), triggering the risk of introduction of exogenous individuals. Nevertheless, fish translocations are increasingly monitored due to international and local regulations, minimizing the potential negative impacts of this limitation. 
Another limitation concerns step 2's experiment. It aims at (i) minimizing expression of aquaculture potential divergences driven by phenotypic plasticity due to specific wild environmental pressures and (ii) allowing observation of those shaped by genetic-based differentiations. Nevertheless, some observed prospective unit performances could not be genetically based and, thus, not be conserved in the next farmed generations. Indeed, even in a common-garden design, population-specific phenotypes can be consequences of past environmental pressures triggering phenotypic plasticity, including epigenetics effects (Massicotte and Angers 2012; Schlichting and Wund 2014) as well as transgenerational effects (Mousseau and Fox 1998; Youngson and Whitelaw 2008). Such phenomena cannot be ruled out since step 2's assessment in based on individuals which (i) parents lived in the wild at least some part of their life or (ii) lived in the wild if eggs are sampled (see step 2's sampling strategies). One solution to minimize this limitation consists in assessing performances over several generations. However, such a solution could be difficult to operationalize for taxa with long lifecycles. Indeed, multigenerations assessment and late sexual maturation species evaluation will increase the workload, the cost, and the length of the approach. However, overall, the use of a common-garden set up allows minimizing the impact of phenotypic plasticity.

\section{Pragmatic concerns}

Our approach can be seen as heavy-going. However, any domestication trials are still time and money consuming. Our approach aims to balance the complexity of multi-trait assessment over several prospective units (though the step 1's classification allows restricting the range of possibilities regarding biological units to compare) by choosing the best part of the wild biodiversity. Indeed, gains of highlighting populations with the highest aquaculture potential can provide important benefits for fish production (see for instance for Perca fluviatilis, Mandiki et al. 2004; Toomey et al. 2019; Vanina et al. 2019a). However, consequent investment may not be operated for each new candidate. One way to decrease investments could consist of extrapolating the knowledge or the results obtained for some species to others in order to minimize the extent of evaluation for new species. Especially, population classification of a species (step 1 when based on genetic differentiation) could be inferred from commonly observed phylogeographic patterns of other co-distributed taxa (e.g. rather than based on a new genetic assessment across the species range (e.g. Danubian regions often host fish populations with genetic specificities and therefore 
could be considered as a relevant place for step 2's sampling). Indeed, an overview of already known phylogeographic structures (e.g. Bernatchez and Wilson 1998; Costedoat and Gilles 2009; Hickerson et al. 2010) allows identifying general phylogeographic patterns in fishes. This means that one could assume that population(s) from a region could be genetically distinct since populations of other taxa display genetic specificities. Therefore, such populations should be evaluated in the step 2.

\section{$\underline{\text { Legal regulation context }}$}

One limitation could be related to national and international regulations regarding the biological material sampling or importation. Indeed, such regulations should be considered before step 2 since it would be a waste of time and energy to perform an assessment on wild populations that could not be imported or sampled to create fish stocks for future production.

\section{WHEN IS THIS APPROACH APPLICABLE?}

The three-step approach is designed to help stakeholders to facilitate domestication programs of new species or species at incipient domestication levels (i.e. from level one to three of the domestication process; Teletchea and Fontaine 2014). At these levels, domestication programs are commonly facing major bottlenecks in domestication and/or production (e.g. Anguilla anguilla; Tomkiewicz et al. 2012). Focusing domestication efforts on the units with the highest aquaculture potential could allow overcoming or minimizing such bottlenecks. Moreover, the approach allows considering all traits important for aquaculture (i.e. multi-trait assessment) from the outset of the domestication programs and thus most likely facilitating future production development.

One could expect that the three-step approach is a less efficient alternative than selective breeding methods that allow improving fish stocks at individual/family levels (Lind et al. 2012). However, selective breeding methods are poorly implementable when only a part of the lifecycle is performed in captivity (i.e. capture-based productions) or when significant number of wild individuals must still be integrated in farmed fish stocks. Under these circumstances corresponding to the early levels of domestication, the three-step approach can be seen as a way to move forward in the domestication process and to design the best fish stocks before potentially handing over to selective breeding programs in the further levels of domestication (i.e. levels four and five). Therefore, the three-step integrative approach and selective breeding methods should be seen as complementary 
rather than opposite and could eventually be used successively along the domestication process. We argue that selective breeding programs should be favored at intermediate stages of domestication (i.e. level four) when the differentiation within population is higher than the interpopulational differentiation (Quillet et al. 2007) or when the genetic gain prediction for one generation of individual selection is higher or equal to the between-population differentiation (Vandeputte et al. 2014). Nevertheless, future studies are needed to assess to feasibility of the threestep approach in the real world production by evaluating costs, gains, and human and technological means. More specifically, cost and benefit comparison with selective breeding programs are needed to assess the complementarity of the two approaches and the relevance of the three-step method. However, cost and benefit analyses are performed for selective breeding programs (e.g. Janssen et al. 2018), but they are not available for the three-step approach since we propose here a new methodology which remains to be tested.

The three-step integrative approach aims to be a model approach applicable to all new candidate species, including non-fish taxa (e.g. for shrimp stripes, Wenger 2012; U.S. Fish and Wildlife Service 2017) provided that the set of traits is adjusted for the target taxon group (e.g. swim bladder inflation or cannibalism rates are not relevant for molluscs while success of successive metamorphoses is). However, as stated previously, the trait choice must also be adjusted according to (i) production goals, (ii) production method, and (iii) technologic advances. First, the approach can be applied to restocking or ornamental market provided that further relevant traits are integrated in category two. For instance, in the case of ornamental aquaculture, external color needs to be considered while resistance to wild pathogens are particularly relevant for restocking programs. Second, the approach adjustment is also necessary when considering some production methods such as polyculture for which traits related to interspecific interactions should be considered. Third, the trait list can also be updated through time as technologic development and new challenges arise (e.g. sperm long-term storage techniques would allow to improve fertilization issues, making fertilization rate less important for the aquaculture potential score; e.g. Alavi et al. 2015; Policar et al. 2019).

\section{WHAT'S NEXT?}

Once the multi-stage aquaculture potential assessment approach is performed, questions can be raised when planning future production. An overview of previous studies which used wild 
intraspecific differentiation at advanced stages of domestication, show that two alternative paradigms to exploit geographic differentiation can be considered (Gjedrem 1985, 2005a): (i) crossing the best prospective units (i.e. crossbreeding) or (ii) concentrating on the best unit to develop production (i.e. purebreeding). On the one hand, crossbreeding aims at exploiting hybrid vigor, or heterosis, which is characterized by offspring performing better than parents for one or several traits. This strategy was explored for several species such O. niloticus (Eknath et al. 1993; Gjedrem 2012) or the rainbow trout (Oncorhynchus mykiss; Ayles and Baker 1983). However, no heterosis effect was found for some species when crossing several wild populations such as $S$. salar (Gjerde and Refstie 1984). This strategy appears as interesting (i) when the aquaculture potential is not that different between prospective units and if an heterosis effect is demonstrated or (ii) when planning selective breeding programs to ensure a large genetic basis. On the other hand, purebreeding, implying a continuous genetic improvement within a biological unit(s) over a long period of time, was also widely used in terrestrial livestock species as well as for fishes (Gjedrem et al. 1991; Gjedrem and Baranski 2009; Gjedrem 2010, 2012). This strategy has been pointed out as particularly efficient when one biological unit presents overall better performances than other units (Gjedrem 1985, 2005a). Indeed, purebreeding has been recommended in breeding programs when there is additive genetic variation while crossbreeding has been suggested if the magnitude of hybrid vigor is larger than the expected genetic gain though a purebreeding program (Gjedrem 1985, 2005a). Whether or not crossing prospective units depends on breeding goals and genetic variation of traits of interest and is beyond the three-step integrative approach which is the first step before potential future breeding programs.

\section{ACKNOWLEDGMENTS}

We are grateful to the European Percid Fish Culture (EPFC) group, the Syndicat Français de l'Aquaculture Marine et Nouvelle (SFAMN) and all fish farmers which contributed to the survey led in this work. LT is supported by a grant from the French Ministère de l'Enseignement Supérieur et de la Recherche. This work was supported by the region Grand Est (DomPop project). 


\section{APPENDICES}

\section{Annex S1: Context of the survey}

The survey focused on two different scenarios targeting species at the beginning of the domestication process and with a high socio-economic interest. The first scenario focused on European production of Percids, taking as an example the European Perch (Perca fluviatilis) and the pikeperch (Sander lucioperca). These two species are among the most promising species to boost European inland aquaculture (Fontaine 2004; Fontaine et al. 2009; Kestemont et al. 2015a) due to their high socio-economic interest (high market value and recreational interest; Kestemont \& Mélard 2000; Kestemont et al. 2015a). They are at their nascent stage of production and geographic differentiation has already been demonstrated for European perch (e.g. Mandiki et al. 2004; Toomey et al. 2019). Percids farming still presents several bottlenecks related for instance to high growth heterogeneity and subsequent cannibalism, low survival rate, or low egg quality (Kestemont et al. 2015a). Therefore, there is a strong interest in improving these species' productions. The second scenario consisted in investigating Sturgeons production (Acipenser ruthenus, Acipenser gueldenstaedtii, Acipenser stellatus, Acipenser baerii, Acipenser persicus, Hybrid bester, and Huso huso) for consumption in Europe. Substantial progress in rearing techniques in the 1980s allowed developing a fruitful sector for meat and caviar production (Bronzi et al. 1999; Williot 2009). The market will most likely extend (Williot 2009), triggering an interest in improving Sturgeon domestication processes. This survey being focused on biological traits, stakeholders involved in the environmental and economic sectors were not consulted in this preliminary investigation. 
The survey was led between February and March 2019. For Percids, between nine and 12 answers were taken into account (variable numbers depending on production goals and production systems of the different farms; e.g. no answers for the larval period when larviculture is not performed in the farm, see Fig. S1 for details), except for anti-predatory behavior for which only three answers were considered since only three farms used outdoor systems. Most of the fish farms use indoor RAS (91.6\% [n=11 farms]), 25.0\% [n=3 fish farms] use outdoor RAS, and two fish farmers use both systems. The fish farms produce fillets ( $75 \%, n=9$ fish farms), fingerlings ( $75 \%, n=9$ farms), and/or wholefish for consumption (33.33\% of the farms, $n=4$ fish farms). Nine farms have more than one production goal. Concerning Sturgeons, between five and eight answers were considered for each trait depending on production goals and rearing systems of the different fish farms (see Fig. S1 for details). Most of the fish farmers use several rearing systems, including flow-through systems (75\% n=6 fish farms), 50\% using indoor RAS ( $\mathrm{n}=4), 25 \%$ using outdoor RAS ( $\mathrm{n}=4)$, and $25 \%$ using ponds $(\mathrm{n}=2)$. Regarding production goals, fish farms produce caviar $(75 \%, \mathrm{n}=6)$, juveniles $(37.5 \%, \mathrm{n}=3)$, fillets $(50 \%, \mathrm{n}=4)$, individuals for restocking $(12.5 \%, \mathrm{n}=1)$, and/or wholefish for consumption $(62.5 \%, \mathrm{n}=5)$. One farm produces Percids and Sturgeons.

Figure S1: Barplots representing the estimation of importance (in \%) for all traits included in the survey for Percids (Perca fluviatilis and Sander lucioperca; graphs on the left column) and Sturgeons (Acipenser ruthenus, Acipenser gueldenstaedtii, Acipenser stellatus, Acipenser baerii, Acipenser persicus, Hybrid Bester, and Huso Huso; graphs on the right column). Barplots represent different group of traits: reproduction period, larval period, on-growing period, traits relative to category 2 [i.e. biological traits which relevance vary according to production systems and production goals], and traits linked to current and future challenges (categories 3 [i.e. biological traits linked to the interaction between the group of individuals and the rearing system, which could impact surrounding environments] and 4 [i.e. biological traits relative to future major challenges and disturbances]). Colors correspond to the different degrees of importance: (i) light grey when the trait was not considered as relevant (even though part of the production goal), (ii) dark grey when the fish farmer has no opinion on the importance, (iii) red when the trait was estimated as negligible, (iv) yellow when the trait was considered as important, and (v) green when the trait was considered as quite important. The number above each trait bar indicates the number of farmers which gave their opinion on this particular trait. 
File available at: $\underline{\text { https://onlinelibrary.wiley.com/doi/abs/10.1111/raq.12430 }}$ 